\title{
Témoignage pour une histoire administrative de la Mission du patrimoine ethnologique
}

An account of the administrative history of the ethnological Heritage Mission at the French Ministry of Culture

\section{Christian Hottin}

\section{OpenEdition}

Journals

Édition électronique

URL : https://journals.openedition.org/insituarss/414

DOI : 10.4000/insituarss.414

ISSN : 2680-4972

\section{Éditeur}

Ministère de la Culture

\section{Référence électronique}

Christian Hottin, «Témoignage pour une histoire administrative de la Mission du patrimoine ethnologique », In Situ. Au regard des sciences sociales [En ligne], 1 | 2019, mis en ligne le 15 octobre 2019, consulté le 20 décembre 2022. URL : http://journals.openedition.org/insituarss/414 ; DOI : https://doi.org/10.4000/insituarss.414

Ce document a été généré automatiquement le 20 décembre 2022.

\section{(c) (i) (2) $\Theta$}

Creative Commons - Attribution - Pas d'Utilisation Commerciale - Pas de Modification 4.0 International - CC BY-NC-ND 4.0

https://creativecommons.org/licenses/by-nc-nd/4.0/ 


\title{
Témoignage pour une histoire administrative de la Mission du patrimoine ethnologique
}

An account of the administrative history of the ethnological Heritage Mission at the French Ministry of Culture

\author{
Christian Hottin
}

1 J'ai exercé, entre 2006 et 2016 la fonction de responsable de l'ethnologie au ministère de la Culture, d'abord en tant que chef de la Mission ethnologie de la direction de l'Architecture et du Patrimoine (Dapa), puis en tant qu'adjoint au chef du département du Pilotage de la recherche et de la Politique scientifique (DPRPS). Un changement de titre, en 2010, qui masque une réforme institutionnelle particulièrement violente pour l'ethnologie (la Révision générale des politiques publiques au ministère de la Culture), mais aussi une remarquable continuité dans les missions qui furent les miennes, avant et après la mise en place de cette nouvelle organisation.

2 Tout au long de ces années, j'ai été amené à rédiger, le plus souvent en vue d'honorer des commandes, de nombreux textes portant sur la politique en ethnologie de la France. Dans la plupart des cas, il s'agissait d'exposer la politique menée en France en faveur de la mise en œuvre de la Convention de l'Unesco pour la sauvegarde du patrimoine culturel immatériel $(\mathrm{PCI})^{1}$. Dans l'écriture de ces textes, j'ai toujours tenu à éviter la posture du témoin ${ }^{2}$, tout autant qu'une forme d'objectivation empruntant la voie de l'écriture historique - une forme de mise à distance qui aurait été possible, l'histoire étant le langage scientifique de ma formation initiale, celle suivie à l'École des chartes. Encore aujourd'hui, je qualifierai tous ces textes de littérature professionnelle, une qualification qui n'a dans mon esprit rien de péjoratif ni de dévalorisant, mais qui me semble simplement correspondre de manière assez pertinente à la situation qui était la mienne au moment de leur rédaction : celle d'un professionnel - du patrimoine, en l'occurrence - exposant vis-à-vis d'autres professionnels (du patrimoine, de l'ethnologie ou du PCI, puisque ce dispositif connut après 2003 une première forme de professionnalisation, bien que très embryonnaire, dans différents pays). L'exemple qui 
m'a guidé, ou à tout le moins qui a souvent été présent à mon esprit en rédigeant ces textes, était celui d'un article lu il y a bien des années maintenant dans La Gazette des Archives, la revue de l'Association des archivistes français. Il s'agit d'un texte consacré à ce qui était alors "un nouveau type d'instrument de recherche", le "répertoire numérique détaillé » (Houriez 1969). De même que ma consœur exposait dans cette contribution un nouveau procédé de description des archives, j'expliquais dans ces articles un nouvel instrument contraignant en faveur de la sauvegarde du patrimoine, la Convention pour la sauvegarde du PCI, et sa déclinaison française. Ce second volet constituait une forme d'expérimentation pratique du dispositif général. Je m'écartais toutefois de mon modèle sur un point : la mise en œuvre concrète n'étant pas pour moi distincte de la description théorique de la Convention, j'étais amené à la contextualiser en décrivant notamment les difficultés rencontrées dans l'assimilation du dispositif de l'Unesco par plusieurs types d'acteurs français: les conservateurs des services du patrimoine, les membres des associations culturelles concernées par le PCI, les ethnologues souvent déroutés par la nouveauté de cette politique. Les réactions des uns et des autres avaient des conséquences sur la conduite ultérieure de la politique, amenaient des inflexions, autant de "frottements", de ce que l'on tient pour négligeable dans les exercices de mécanique proposés aux lycéens en cours de physique, mais qui, dans la mise en pratique, pouvaient amener des évolutions importantes à moyen terme.

\section{A Filetta, groupe de cantu in paghjella.}

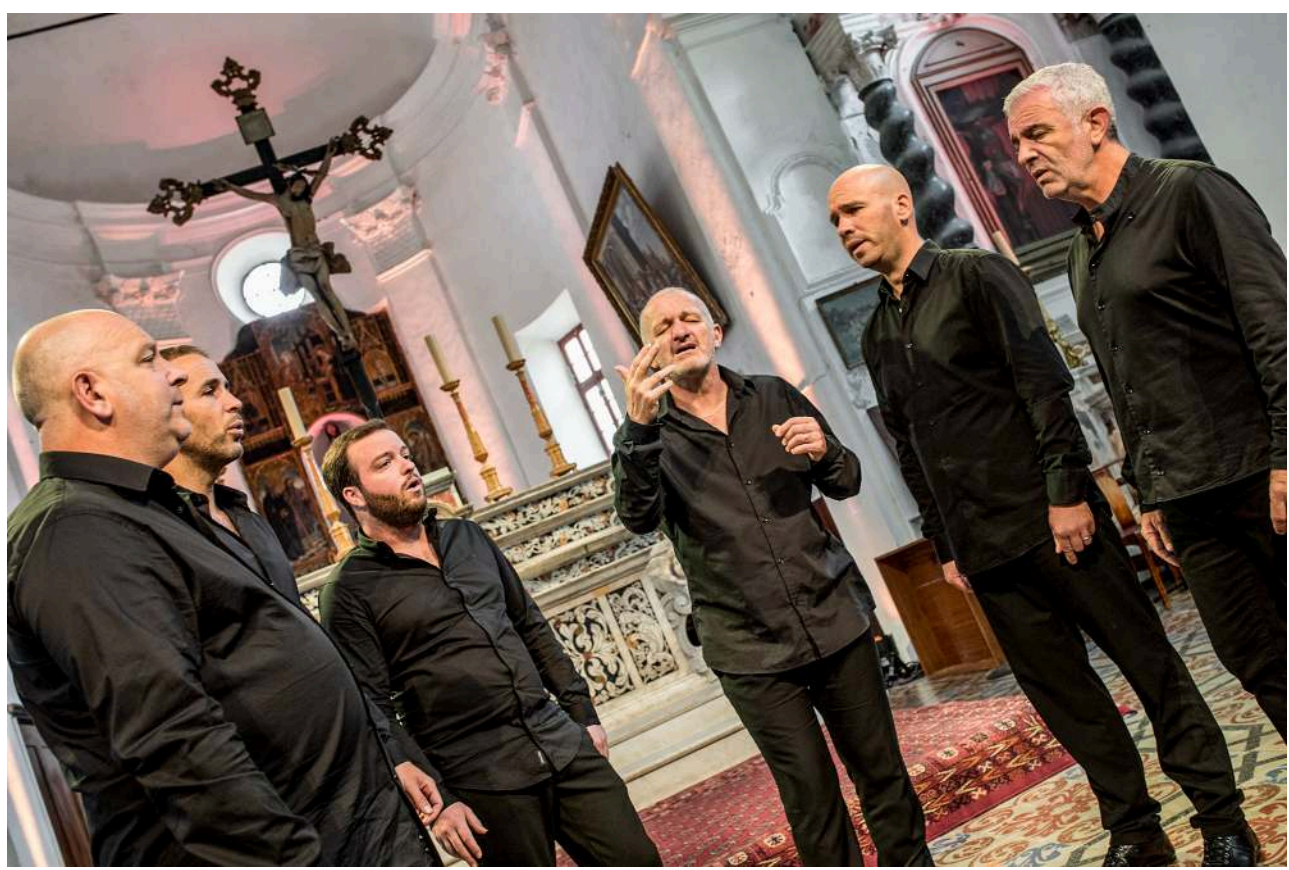

Inscrit en 2008 sur la Liste de sauvegarde du patrimoine culturel immatériel de l'Unesco, le cantu in paghjella corse fait partie des premiers éléments français présenté sur ces listes internationales.

(c) Armand Luciani / A Filetta.

3 À contre-courant de ce qui fut mon positionnement pendant ces années, j'assumerai ici tout à la fois la posture du témoin - une bonne fois pour toutes, en ne désirant plus m'exprimer par la suite sur cette question - et celle de l'historien, moins pour écrire cette histoire de la Mission du patrimoine ethnologique, du reste, que pour esquisser 
diverses pistes utiles à emprunter, selon moi, en vue d'écrire cette histoire, une histoire qui serait ni plus ni moins utile à écrire que celle d'une autre organisation. Un propos qui n'appelle aucune forme de comparaison, et encore moins d'opposition, avec une possible anthropologie "pragmatique et plébéienne" issue du patrimoine ethnologique. Ayant quitté le service de l'ethnologie au ministère depuis maintenant plus de deux ans et ayant entamé depuis d'autres travaux, je peux estimer le temps venu de tirer symboliquement un trait sur ce que furent ces douze années de travail.

\section{Une équation complexe : être chef de la Mission ethnologie au temps de la RGPP}

\section{De quelques dates importantes pour l'histoire du patrimoine ethnologique}

4 Je n'ai pas été le témoin oculaire de toute l'histoire du patrimoine ethnologique, loin s'en faut. Il me semble, rétrospectivement, que certaines dates ont particulièrement compté pour ses acteurs principaux, les ethnologues du ministère de la Culture: 1995-1996 notamment, qui correspond à la plus grande extension du réseau territorial, au début des ethnopôles et à l'amorce du «tournant réflexif » de la Mission, pour reprendre l'expression de Jean-Louis Tornatore (2004) ; 2000-2001 ensuite, qui est tout à la fois le temps de la fin du Conseil du patrimoine ethnologique et celui du lancement du Laboratoire d'anthropologie et d'histoire de l'institution de la culture (Lahic), soit l'institutionnalisation de ce tournant réflexif en administration centrale. J'y ajouterai enfin 2006, avec l'arrivée du PCI, compte tenu de l'émoi que cette Convention a suscité dans notre modeste milieu.

5 Cette chronologie sommaire de l'histoire de la Mission paraît relativement déconnectée de l'histoire générale de l'administration du patrimoine dans la France contemporaine. Prenons là encore deux dates: 1990, la création du corps des conservateurs du patrimoine et la mise en place de l'École nationale du patrimoine (ENP, future INP); 2003, le rassemblement des différentes lois sectorielles sous le nom de code du patrimoine. De ces deux entreprises d'unification, l'ethnologie est absente, alors qu'elles tendent l'une comme l'autre à définir le périmètre de l'action publique patrimoniale (archives, musées, monuments historiques et inventaire général, archéologie, mais non les bibliothèques, absentes de l'ENP et marginales dans le code). Là encore, j'ajouterai une troisième date, 2010, celle de la mise en place de la direction générale des Patrimoines, une organisation qui confirme et consolide ce périmètre, et qui, une fois encore se met en place sans l'ethnologie, puisque symboliquement celle-ci est mise en congé de l'organigramme du ministère. 
Visite patrimoniale.

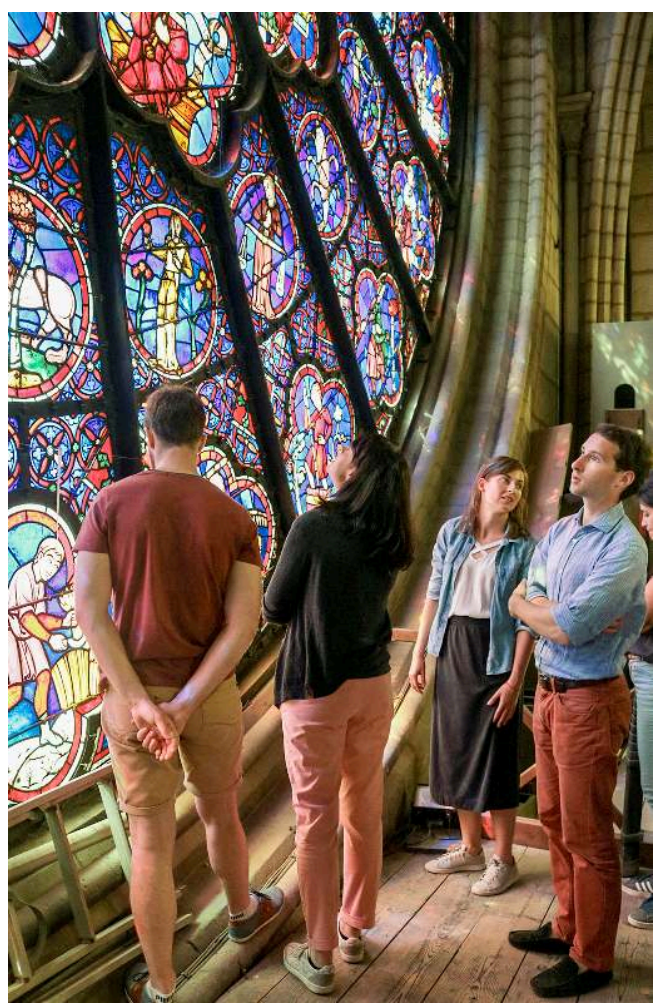

La formation des conservateurs du patrimoine se décline en cinq spécialités : archéologie, archives, Monuments historiques-Inventaire, musées et patrimoine scientifique technique et naturel (PSTN). L'ethnologie n'en fait pas partie.

(C) Patrick Dumeix / cliché INP.

Enfin, deux dernières dates pourraient être regardées avec dédain tant elles paraissent relever de la petite histoire administrative, celle des guerres picrocholines de chefs de bureau. Elles concernent des événements qui, sans trop modifier, en apparence, sa situation, ont eu en fait un lourd impact sur le devenir de la Mission du patrimoine ethnologique. En 1997, est créée la direction de l'Architecture et du Patrimoine (Dapa), déplaçant le centre de gravité de l'institution du patrimoine vers la production et la conservation du bâti (et donc à moyen terme marginalisant, dans les débats et les prises de décision, une politique qui ne s'y rattache en rien). En 2005, enfin, la sous-direction de l'Archéologie, de l'Ethnologie, de l'Inventaire et du Système d'information (Archétis) s'installe dans l'immeuble parisien des Bons-Enfants, réunissant en un même lieu l'archéologie, l'ethnologie et ce qui subsiste de l'Inventaire général après la décentralisation. Un moment traumatisant pour les membres de cette dernière institution, la décentralisation étant vécue comme une forme de déclassement du dispositif d'action; un moment aussi de recherche des convergences, notamment à travers le développement du recours à la notion de "contrôle scientifique et technique » : on se rendit compte alors que l'ethnologie peinait lourdement à s'insérer dans ce cadre.

7 J'adopterai donc un ton plus personnel pour évoquer ces quatre années de vie de la Mission ethnologie à la Dapa. Recruté comme chef de la Mission en octobre 2005, j'ai commencé à travailler au sein de la sous-direction Archétis en janvier 2006. Je n'avais jamais entendu parler du patrimoine culturel immatériel avant de prendre mes fonctions et mon attrait pour l'ethnologie était essentiellement motivé par les 
recherches du Lahic, que j'avais découvertes quelques années plus tôt lors du colloque «Le lieu de l'archive » organisé à l'École des hautes études en sciences sociales (EHESS) en juin 2003. Considérée isolément ou dans ses relations avec ses partenaires, la Mission rencontrait alors un certain nombre de difficultés.

\section{L'ethnologie, combien de divisions?}

8 Les relations avec le Lahic, auquel étaient dès cette époque rattachés de nombreux ethnologues de la Mission ou des directions régionales des Affaires culturelles (Drac), étaient au point mort, en raison des tensions survenues en 2005 entre Odile Welfélé, la précédente chef de la Mission, et le directeur du Lahic, Daniel Fabre. D'autre part, cette unité mixte de recherche (UMR), qui avait été la concrétisation d'une réorientation majeure de la politique de l'ethnologie en 2000, encouragée par Jean-Marie Jenn, alors chef de la Mission, ne suscitait plus aucun intérêt de la part de la direction de l'Architecture et du Patrimoine, alors même que des moyens humains et budgétaires importants avaient été mis au service de ce nouveau dispositif. Les relations avec le réseau des conseillers pour l'ethnologie étaient également affaiblies, puisqu'ils n'avaient pas été réunis depuis plus d'un an. Leur «remonter le moral», selon les termes mêmes d'Isabelle Balsamo, sous-directrice chargée de l'Archéologie, de l'Ethnologie, de l'Inventaire et des Systèmes d'information, faisait partie de mes objectifs.

$\mathrm{Au}$ sein même de la sous-direction, le pilotage de la politique de l'ethnologie était compliqué par la répartition de l'équipe entre deux bureaux. D'une part, la Mission proprement dite avait la charge du réseau des conseillers, de la recréation d'un Conseil du patrimoine ethnologique et de la question du PCI («essentiellement du jus de cervelle») De l'autre le département Recherche, Méthode et Expertise regroupait plusieurs ethnologues appartenant à la Mission avant la réorganisation. Créé à partir de et sur le modèle du bureau de la Méthode de l'ancienne sous-direction de l'Inventaire, il rassemblait en son sein un "collège d'experts » issus de l'archéologie, des différents champs de compétence de l'Inventaire et de l'ethnologie. Les publications, et notamment la revue Terrain, totalement dissociée de la Mission, se trouvaient également dans ce département, qui avait pour fonction principale la conception et le pilotage de programmes scientifiques - soit le cœur de métier historique de la Mission. 

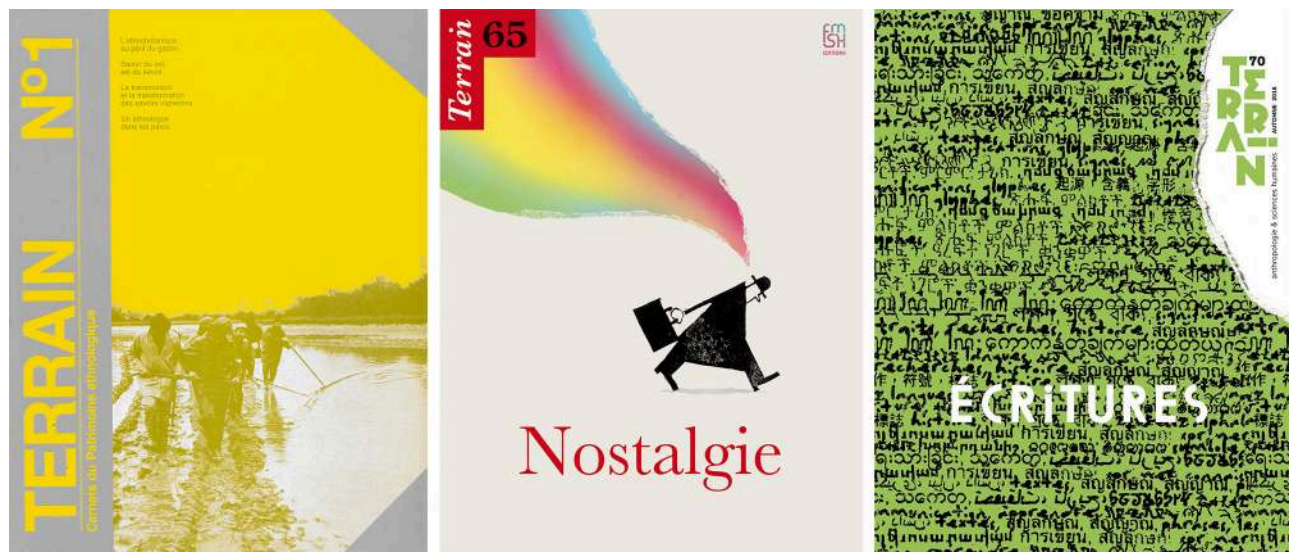

Depuis les « Carnets du patrimoine ethnologique » jusqu'à « Anthropologie et sciences humaines » en passant par «Revue d'ethnologie de l'Europe » : histoire de l'autonomisation d'un projet éditorial ?

(C) ministère de la Culture

10 L'ethnologie avait joué dans cette réorganisation le rôle de variable d'ajustement. Son éclatement rendait difficile la construction d'une politique cohérente et nuisait à sa visibilité; elle était en effet prise en étau entre l'Inventaire général, en quête d'un nouveau mode de gouvernance de son réseau désormais décentralisé, et l'archéologie, portée par une forte dynamique qu'appuyait directement le directeur de l'Architecture et du Patrimoine, notamment à travers le développement du programme des centres de conservation et d'études (CCE).

\section{La conduite de la RGPP au sein de la Dapa, du point de vue de Fabrice à Waterloo}

11 Cette recherche d'équilibre, qui était commune aux trois composantes de la sousdirection (archéologues, ethnologues, personnels de l'Inventaire), allait bien vite être bouleversée par la mise en place de la Révision générale des politiques publiques. À cet égard, deux traits méritent d'être soulignés pour caractériser sa mise en œuvre au sein du ministère de la Culture : d'une part, sa durée, plus importante que dans d'autres institutions (plus de deux ans), et, d'autre part, son pilotage en interne, sans recours à un cabinet d'audit et de conseil, le directeur de l'Architecture et du Patrimoine, Michel Clément, ayant été choisi comme préfigurateur de la future direction générale des Patrimoines, destinée à réunir musées, archives, architecture et patrimoines.

Avec le recul que donnent les quelque huit années passées depuis son achèvement, force est de reconnaître que la RGPP n'a en rien constitué un "coup de rabot» uniforme sur l'administration centrale, et resta très éloignée, en ce sens des «commissions de la Hache» ou «de la Guillotine» de 1947 ou 1949³. Certaines composantes du patrimoine en sont sorties renforcées (archéologie, Monuments historiques); certaines sont parvenues à maintenir peu ou prou leur périmètre de compétence (musées), d'autres enfin en sont sorties affaiblies. Les Archives de France, de manière dramatique, et l'ethnologie ont été de celles-là. Pour l'ethnologie, le diagnostic fut posé, dès janvier 2008, en réunion de sous-direction, avant même que ne soient mis en place des groupes de travail de préfiguration: il serait extrêmement difficile de conserver une mission autonome à l'issue du processus. En tant que chef de 
la Mission en question, je n'ai jamais souscrit à ce diagnostic, qui, dans les faits, s'est avéré correspondre à la décision prise par la direction du Patrimoine, et notamment par son directeur agissant alors en tant que préfigurateur de la future direction générale.

La suite du processus a fait émerger - sans aucune concertation à aucun moment avec l'équipe de la Mission ethnologie - le rattachement de celle-ci au projet de département du Pilotage de la recherche et de la Politique scientifique. Un rattachement ouvertement présenté par le préfigurateur de la direction générale des Patrimoines comme une "évaporation", selon les termes de Michel Clément. Concrètement, l'inclusion des missions de l'ethnologie dans ce département transversal était pertinente par rapport à toute l'histoire antérieure de la Mission, qui avait été préfiguratrice en matière de pilotage de la recherche (notamment à travers sa politique d'appels d'offres pour le lancement de programmes de recherche). En revanche, cette incorporation dans un service transversal était nettement plus problématique pour traiter efficacement la question du PCI, politique de sauvegarde et non de recherche. S'agissant de ce versant de l'action de la Mission, différentes pistes furent explorées, telles que le rattachement au service des Affaires internationales ou l'insertion au sein de la Mission Inventaire général, option qui constitua un véritable casus belli aux yeux de l'équipe de la Mission ethnologie. Finalement, c'est bien l'ensemble des missions de la Mission, PCI compris, qui furent rattachées au DPRPS.

Lors d'une réunion des conseillers pour l'ethnologie, la sous-direction Archétis présenta la fin de l'autonomie de la Mission comme une perte qui devait être compensée par un gain: la création, sur des bases réglementaires, d'une nouvelle instance de pilotage, un Conseil du patrimoine ethnologique et immatériel, qui, utilisant le décret de 2005 réformant le Conseil du patrimoine ethnologique, lui donnerait un second souffle en le posant comme organisme de gouvernance de la politique du PCI. Le Secrétariat général du Gouvernement (SGG) était justement en train de procéder à un passage en revue de l'ensemble des instances de consultation de l'État, avec pour objectif de trier celles qui devaient être supprimées, conservées ou modifiées. Pour le Conseil du patrimoine ethnologique et immatériel, une étude de nécessité et un projet furent rédigés, dont la direction du Patrimoine loua la qualité. Las, lorsque le SGG rendit son verdict, le conseil en question ne figurait ni dans les commissions à supprimer, ni dans celles à conserver, et pas davantage au nombre de celles à réformer. Il apparut tout simplement que le dossier n'avait pas été transmis. Aucune explication ne fut jamais fournie sur cet « oubli ».

En janvier 2010, la direction générale des Patrimoines se mit en place, sans service dédié à l'ethnologie ni instance de gouvernance de cette politique, mais avec un mandat clair sur le pilotage de la convention de l'Unesco.

\section{Notes pour une histoire positiviste de la Mission du patrimoine ethnologique}

16 Le témoignage qui précède, avec ses lacunes (j’ai volontairement laissé de côté la période la plus récente, postérieure à l'achèvement de la RGPP), peut être de nature à modifier ou à éclairer la lecture des articles rédigés pour rendre compte des actions conduites dans le domaine de l'ethnologie au cours de cette période, notamment dans le champ du patrimoine culturel immatériel. Il est, en soi, insuffisant pour constituer 
une histoire de la Mission du patrimoine ethnologique dans la dernière période de son existence autonome. Plus généralement, une histoire du patrimoine ethnologique reste à écrire, qui ne pourrait l'être que par un historien n'ayant pas eu part au dispositif, ce qui n'est pas le cas de l'ancien chef de la Mission, pas plus que celui des ethnologues qui s'y sont essayés. Les uns comme les autres ont trop partie liée au dispositif pour l'appréhender de manière non partisane.

prendra un seul exemple: la théorisation et la réflexion rétrospective sur le "tournant réflexif » de la fin des années 1990, menées par ceux qui en furent euxmêmes les acteurs, laissent de côté le fait que ce renversement de paradigme ne fut en fait opéré que par une partie seulement des ethnologues de la Mission ou des directions régionales, les autres continuant peu ou prou à se reconnaître dans le cadre préexistant $\mathrm{du}$ " patrimoine ethnologique ». Il me semble que ce sont aussi ces derniers qui se sont par la suite le plus aisément saisis du patrimoine immatériel. Ils y trouvèrent, en effet, avant tout des formes de continuité avec la politique antérieure et furent de ce fait aptes à s'emparer de l'ensemble de ce nouveau dispositif (inventaire et candidatures compris), sans devoir, comme les adeptes du tournant réflexif, opérer une lecture sélective de la Convention (centrée sur la participation, mais minorant l'objectif de sauvegarde) et une purification de la notion pour la rendre compatible avec leur posture.

18 À rebours d'une perspective surplombante et hautement sélective sur l'histoire de la Mission, attentive avant toute chose aux débats théoriques qui agitèrent le landerneau ethnologique du ministère, et oublieuse parfois de l'œuvre concrète et pratique de certains collègues en région, on défendra ici l'idée qu'un tel projet relève de la meilleure tradition de l'histoire administrative française, celle défendue et illustrée par Guy Thuillier dans ses recherches et ses enseignements professés à l'École pratique des hautes études (IVe section, sciences historiques et philologiques). L'histoire des bureaux, des services, l'histoire des institutions administratives en général, volontiers épaulées par les comités d'histoire des ministères, ne jouissent pas dans le milieu des historiens d'une cote d'amour élevée. Plane sur elle la suspicion d'être une « histoire maison» et non une simple histoire «de la Maison». Espérons que le patrimoine ethnologique saura trouver son historien, avec ou sans le soutien du Comité d'histoire du ministère de la Culture. C'est à lui que nous destinons les quelques jalons qui viennent conclure ce témoignage.

\section{Quelles sources?}

19 En 2009, alors de la RGPP suivait son cours, la Dapa forma le projet d'élargir le périmètre de collecte de la Médiathèque de l'architecture et du patrimoine, alors circonscrit principalement aux Monuments historiques, à tous les services de la direction. S'il avait été mené à son terme, ce projet aurait conduit la Mission du patrimoine ethnologique à remettre elle aussi ses archives définitives à la Médiathèque. Que la direction en question fût appelée, sous peu de mois, à se dissoudre dans un ensemble plus vaste, au sein duquel de surcroît se trouvait la direction chargée du pilotage de la politique archivistique pour l'ensemble de l'État, ne semblait pas poser de problème. Je m'élevais à l'époque avec virulence contre ce projet pour ce qui concernait les archives de l'ethnologie, rappelant que la Mission du patrimoine ethnologique, depuis son origine, avait effectué, en relation avec la Mission des archives de France au 
ministère, de nombreux versements aux Archives nationales, ainsi qu'il est normal que toute administration centrale le fasse.

Archives nationales, site de Pierrefitte-sur-Seine.

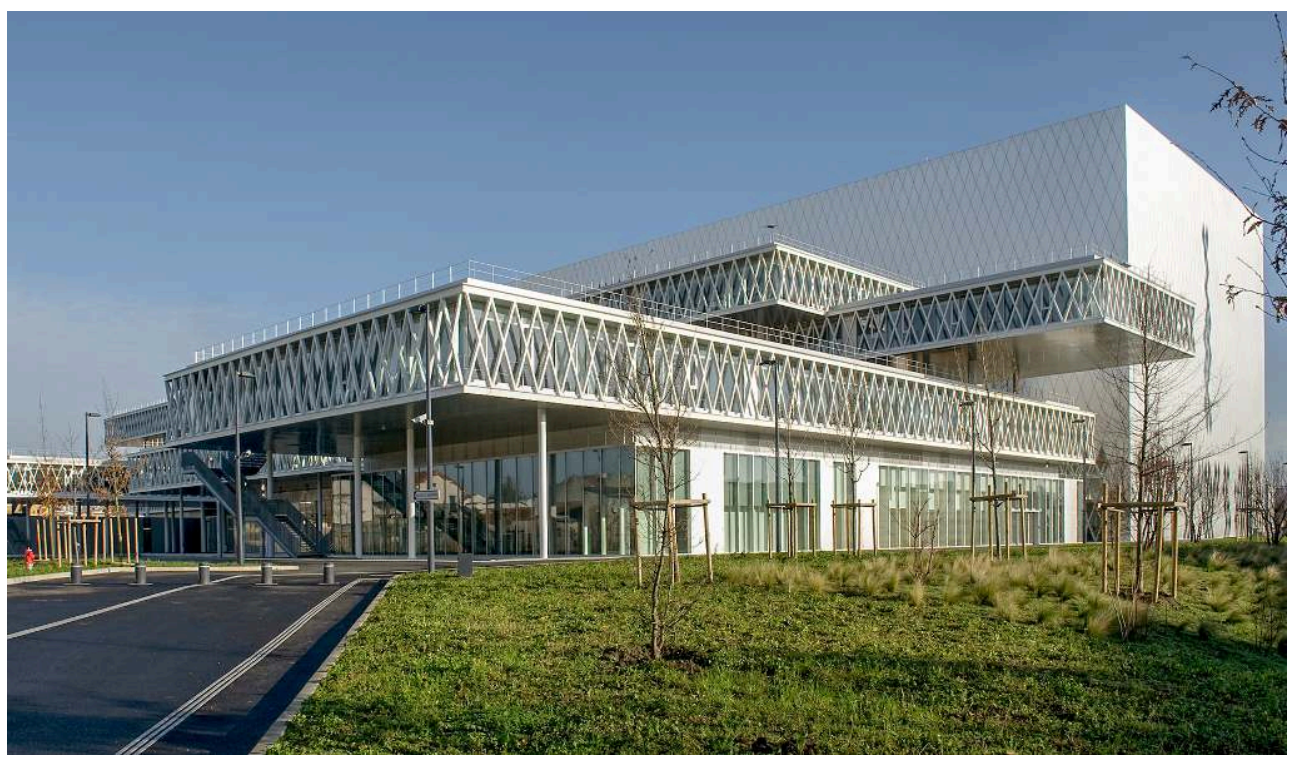

Les administrations centrales de l'État versent, via les missions implantées dans les ministères, leurs archives définitives aux Archives nationales. Le secteur du patrimoine fait largement exception, mais pas la Mission du patrimoine ethnologique.

(c) Alain Berry / cliché Archives nationales.

De fait, les sources archivistiques de l'histoire de la Mission sont aisément accessibles. Encore faudrait-il qu'elles soient croisées avec celles de la Dapa, dont elle relevait et qui fut au cœur du processus de décision pour les réformes ayant l'incidence la plus directe sur la vie de la Mission: celle de 2004, qui mit en place le rapprochement avec l'archéologie et l'Inventaire, en même temps que l'éclatement en deux de la Mission (publications d'un côté, pilotage du réseau de l'autre), et celle de 2009, qui déboucha sur la naissance du DPRPS. Des entretiens oraux avec les directeurs et sous-directeurs concernés, Michel Clément et Isabelle Balsamo, seraient certainement fructueux.

\section{Une analyse budgétaire, une analyse de la gouvernance et des stratégies}

21 La mise en œuvre d'une fongibilité des crédits des différents services qu'avait incorporés la sous-direction Archétis (Archéologie, Ethnologie, Inventaire et Systèmes d'information) rendait particulièrement ardue l'identification des lignes dévolues à l'ethnologie. Si l'érosion de ces crédits sur la longue durée semble difficilement contestable, faute d'ancrage solide des actions dans des missions garanties par le droit, elle reste à quantifier... C'est en corrélation avec cette analyse budgétaire que pourra être conduite une analyse des modes de gouvernance de la Mission du patrimoine ethnologique (rôles respectifs des vice-présidents du conseil, des directeurs de l'Architecture et du Patrimoine et des chefs de la Mission) et que pourra être élaborée une histoire reposant de manière moins univoque peut-être sur le basculement du « tournant réflexif » des années 1996-1997. 


\section{Une approche comparée}

À la fin des années 1970, les univers socioprofessionnels de l'archéologie, de l'ethnologie et de l'Inventaire général présentaient, semble-t-il, un certain nombre de traits communs : forte présence du monde associatif, statuts dans l'emploi public très divers aussi, possibilités de professionnalisation dans l'administration culturelle qui offraient une alternative aux débouchés très restreints disponibles offerts dans ces mêmes secteurs par l'institution académique. L'échec de la professionnalisation de l'ethnologie (Rautenberg 2008) fait contraste avec les réussites enregistrées par l'archéologie et, dans une moindre mesure, par l'Inventaire, la décentralisation de 2004 ayant conduit à une remise en cause des fondements de cette institution très pluridisciplinaire, moins identifiée que les deux autres à une communauté de métier.

Cet échec questionne en creux le succès des autres politiques: le succès de l'archéologie, à cet égard, est sans doute moins celui du patrimoine archéologique et de la valeur qu'on lui attribue, fût-elle consacrée par la loi, que celui des archéologues, rompus à l'action collective (parce que voués, dans l'exercice de leur discipline scientifique, au travail collectif?) et capables de s'organiser pour atteindre leurs objectifs. Alors même que les ethnologues, « communauté de solitaires " selon la belle expression de Gérard Lenclud, peinaient à faire de même....

Monique Jeudy-Ballini chez les Sulka (Papouasie-Nouvelle-Guinée), 1984.

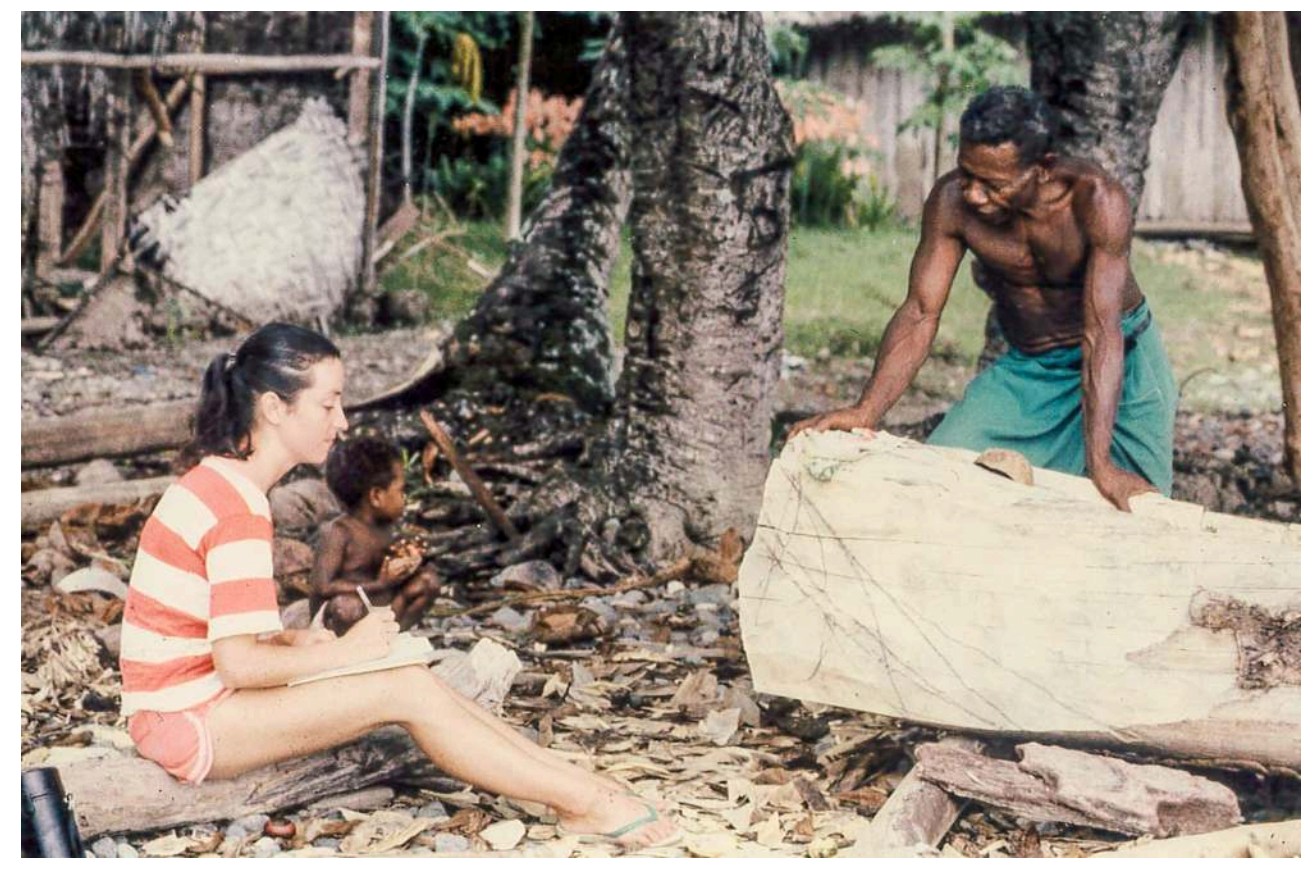

Les ethnologues : une communauté de solitaires... en dialogue constant avec le monde.

(c) Jacques Jeudy.

\section{Une histoire spatiale de la MPE}

De la rue de la Banque à la rue des Pyramides, en passant par la rue de Richelieu, la rue Auguste-Vacquerie et la rue Saint-Honoré, la Mission a connu entre 1980 et 2010 pas 
moins de cinq déménagements. Ce nomadisme ne lui est pas propre, elle le partage avec nombre d'autres services du ministère de la Culture, institution dont la géographie et l'organigramme ont toujours été fâchés. Il est néanmoins particulièrement prononcé dans le cas de l'ethnologie. Anecdotique en apparence, une telle étude a néanmoins du sens pour qui veut comprendre l'accès à l'information (notamment informelle) dont pouvaient bénéficier les agents de la Mission pour tout ce qui relevait de la vie de la DP puis de la Dapa, de même qu'elle apporte des indications tant objectives que subjectives sur la position du service par rapport au centre de décision de l'institution (le bureau du directeur). À ce titre, l'installation rue Auguste-Vacquerie, au début des années 2000, semble avoir été clairement vécue par l'équipe de la Mission comme une relégation annonciatrice des pires difficultés.

Immeuble des « Bons-Enfants », Paris.

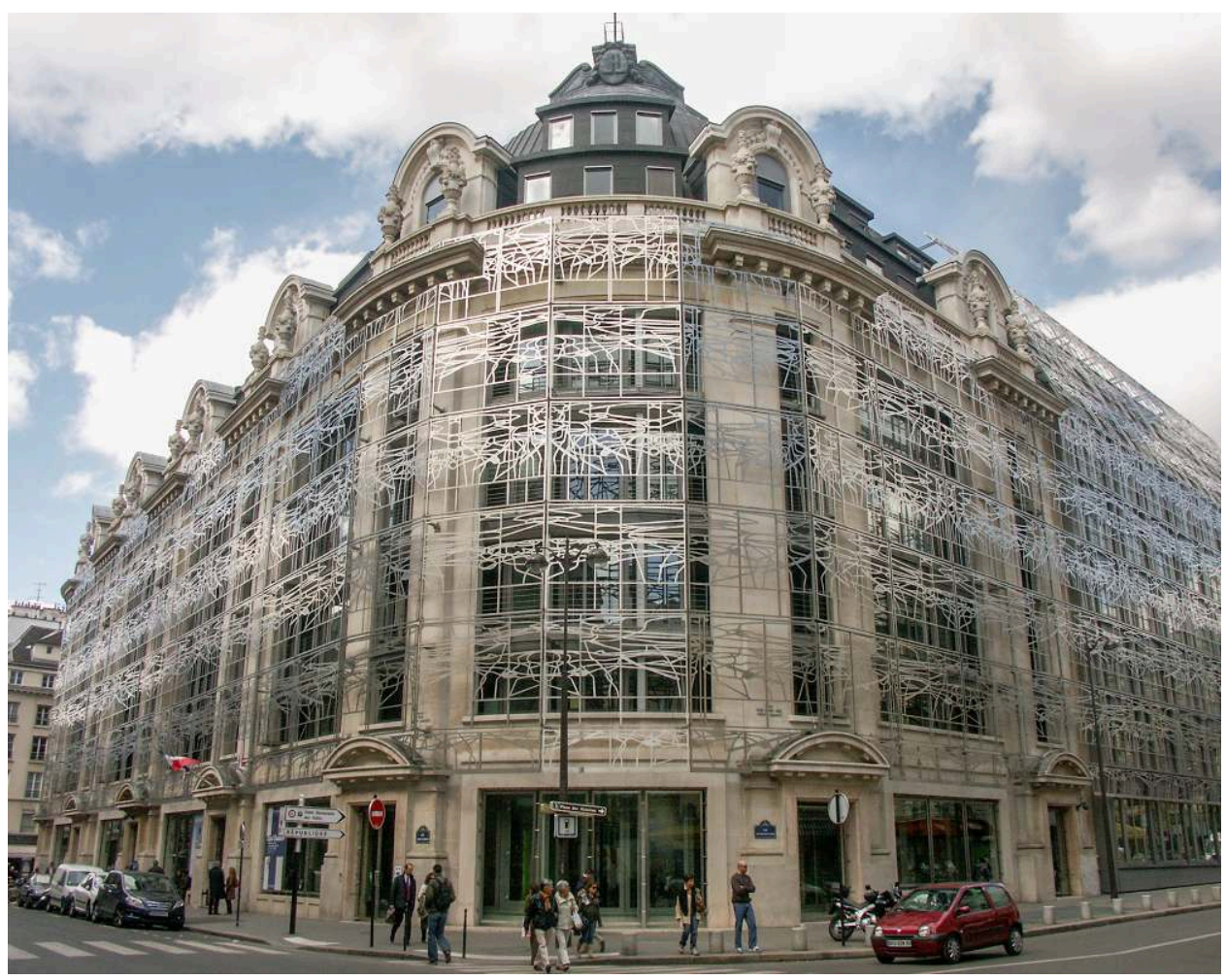

Le site principal de l'administration centrale du ministère de la Culture est l'un des nombreux visages du nomadisme de l'ethnologie au ministère, entre 2005 et 2010.

(c) cliché ministère de la Culture, DCOM, DGPAT.

\section{Quels chefs pour la Mission du patrimoine ethnologique?}

Volontiers centrées sur la figure des vice-présidents du Conseil du patrimoine ethnologique (Isac Chiva, Marc Augé, Daniel Fabre, Christian Bromberger) (Barbe 2013), les analyses de la politique conduite par la Mission du patrimoine ethnologique ont largement laissé dans l'ombre le rôle tenu par les chefs de la Mission, à l'exception notoire de la première titulaire du poste, Élizabeth Fleury. Il serait tout à fait possible, et sans doute très intéressant, de constituer un corpus d'archives orales à partir de l'enregistrement des témoignages de ces chefs de la Mission, puisque, à l'exception du regretté Jean-Marie Jenn, tous sont encore vivants aujourd'hui. Leur parole serait 
notamment d'une grande aide pour comprendre les relations entre la Mission et la DP, puisque, en tant que chefs du service, ils devaient porter la politique de la Mission devant le directeur du Patrimoine puis celui de l'Architecture et du Patrimoine. Elle serait également précieuse pour éclairer d'un autre regard la conception que les viceprésidents successifs avaient de leur rôle. Elle serait du plus haut intérêt pour comprendre la perception que les uns et les autres ont eu de la diversité des postures des ethnologues en région, voire de leurs dissensions éventuelles. Elle serait enfin passionnante pour savoir comment ils ont appréhendé un domaine dont ils ignoraient tout, un métier qui n'était pas le leur, une discipline qui leur était étrangère de par leur formation initiale.

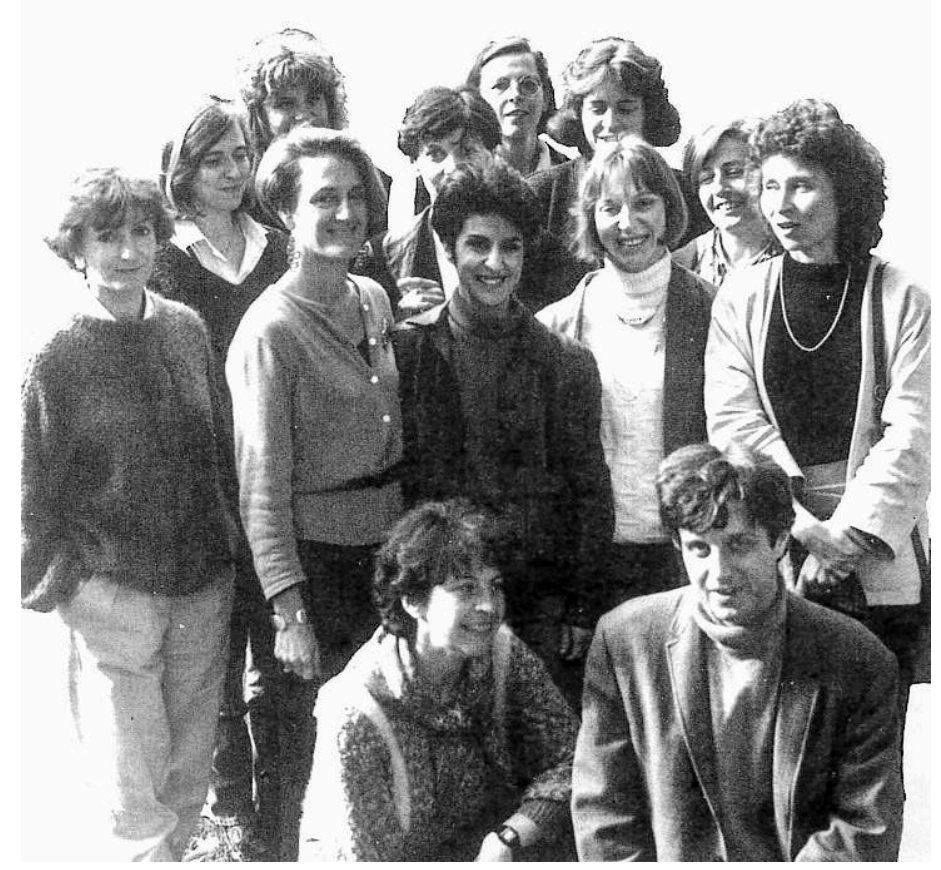

Pour une histoire vivante du patrimoine ethnologique : l'équipe de la Mission du patrimoine ethnologique autour de sa première chef Élisabeth Fleury (tout à droite), entre 1980 et 1989. (c) Christian Voinet / cliché ministère de la Culture, DPRPS ; DGPAT.

En effet, et ce ne sera pas un moindre défi pour cet historien de la Mission, il lui faudra tenter de rendre compte de cette étrangeté, celle d'une Mission du patrimoine ethnologique qui ne fut jamais dirigée par un ethnologue, mais, après 1989, par des chartistes, historiens de formation sinon de pratique, et quasiment tous, en dehors de Michel Melot, archivistes de métier. On pourrait tenter de comprendre, au-delà des réponses préfabriquées sur les affinités supposées entre ethnologie et archives, ce qui a poussé ces femmes et ces hommes à faire un "pas de côté " dans leur carrière d'archiviste, comment ce passage à la Mission s'est inscrit dans une trajectoire professionnelle (et à quel moment de celle-ci), comment enfin ils ont négocié leur sortie de la Mission après un temps plus ou moins long passé à sa tête. 


\section{Une prosopographie des ethnologues de la Mission}

Une telle entreprise apparaît largement faisable, eu égard au nombre relativement restreint de personnes concernées. Il importerait de connaitre leur parcours académique avant leur entrée au ministère de la Culture, les raisons de leur venue en son sein, leur parcours professionnel (en administration centrale comme en direction régionale), ainsi que, éventuellement, leur parcours après être passé par la Mission.

Une autre caractéristique du personnel de la Mission ethnologie est en effet, outre la présence systématique de responsables étrangers à la discipline, la très forte mobilité de ses agents vers l'extérieur, que ce soit pour rejoindre en détachement ou en mise à disposition un laboratoire du CNRS ou pour évoluer vers des corps de recherche (maître de conférences, chargé de recherche) ou scientifique (conservateurs du patrimoine). Ce mouvement semble s'être accéléré à partir de la fin des années 1990, peu de temps après que la Mission a atteint ses effectifs les plus importants, et son réseau régional sa plus grande extension. Indubitablement, une telle volatilité pose question. Toutes ces étapes préparatoires (étude des archives administratives, constitution de corpus d'archives orales, entretiens, constitution d'une prosopographie du personnel) permettront d'écrire une histoire de la Mission du patrimoine ethnologique en proposant une chronologie nouvelle de l'existence de cet objet administratif bien singulier.

\section{En région comme à Paris, une histoire déconcentrée et décentralisée de l'ethnologie}

Une étude prosopographique de l'ensemble des ethnologues liés à l'histoire de la Mission ouvre naturellement la voie à un autre chantier tout aussi considérable : celui de l'implantation de la politique du patrimoine ethnologique dans les régions. Ce point a été relativement peu abordé dans la première partie de notre propos, centré sur un témoignage de la vie de la Mission au sein de l'administration centrale. Il est essentiel. En effet, tout au long de son histoire, la Mission ethnologie (ses chefs, ses équipes) a eu à se poser la question de l'articulation entre son rôle d'administration centrale et son action territoriale.

Cette question recouvre des aspects budgétaires (on pense par exemple aux crédits de programmes de recherche en région, perdus par la Mission dans les années 1990) tout autant que des aspects humains (on songe ici à la croissance continue des effectifs de conseillers dans les directions régionales, entre 1980 et 1995, avant que ces effectifs ne stagnent puis ne décroissent régulièrement). Elle est avant tout d'ordre stratégique : quelle organisation de l'ethnologie sur le territoire, pour quelle politique?

Pour ce qui est de l'organisation, il suffira de rappeler ici que plusieurs modalités d'implantation ont été successivement et concomitamment mises en œuvre: l'internalisation au sein des directions régionales (avec des conseillers sectoriels), la contractualisation avec les collectivités territoriales (on fait ici référence à la création du Crécet en Basse-Normandie en 1984, avec la tête de cette "Mission ethno normande " un directeur qui participait aux réunions de chefs de service en Drac) et enfin l'externalisation, à travers la mise en place d'un réseau de structures de toutes natures labellisées "ethnopôle " au titre de leur coopération avec le ministère. Au fil du temps, ces différentes modalités d'action ont pu se superposer ou au contraire se 
succéder: le binôme "conseiller pour l'ethnologie-ethnopôle" fut longtemps présenté comme la garantie d'une action équilibrée, avant que ne soient créés des ethnopôles directement reliés à la Mission dans des régions dépourvues de conseillers ; le Crécet, quant à lui, est devenu ethnopôle dans les années 2010, à la faveur de sa mutation en établissement public de coopération culturelle sous le nom « Fabrique des patrimoines en Normandie ».

Affiche du festival « Altérités », 2019.

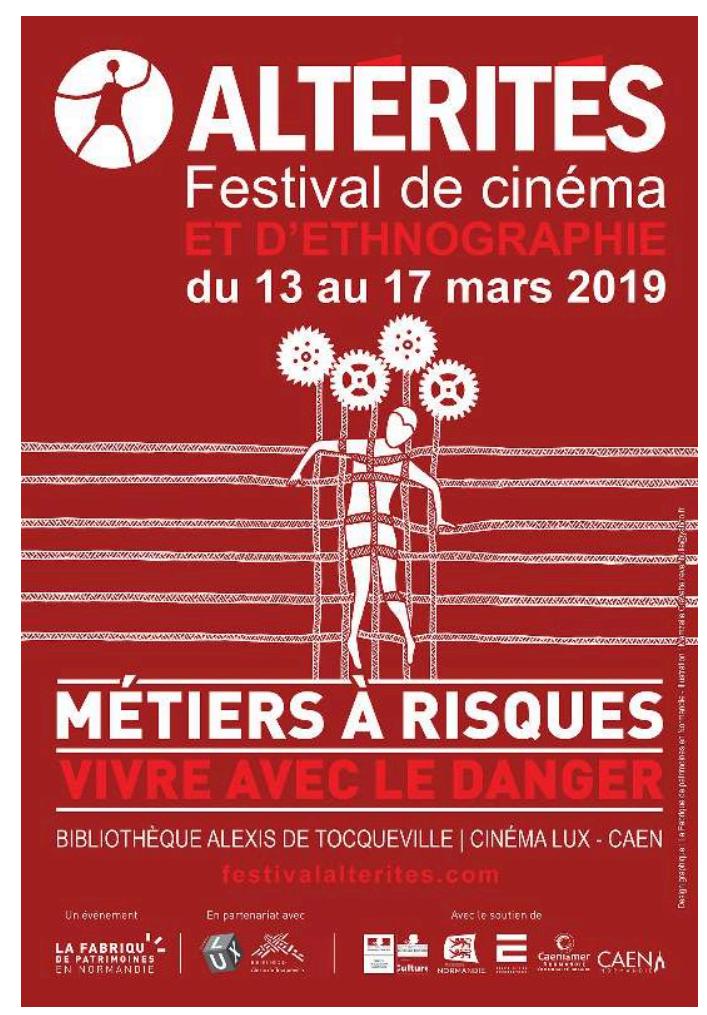

Le Crécet est un exemple atypique de l'ethnologie pratiquée en régions. Initialement conçu comme une « Mission du patrimoine ethnologique en région », il se nomme désormais « Fabrique des patrimoines en Normandie», et propose notamment un festival du film ethnographique.

(c) Mamzelle Crevette Revenbulle@yahoo.fr / cliché La Fabrique des patrimoines en Normandie.

S'agissant de la politique conduite, tous les acteurs concernés s'accordent pour dire mais ce point devrait être étudié en détail et sans doute nuancé - que l'ethnologie en région a pris, du fait de sa proximité avec le terrain associatif et avec le monde des collectivités territoriales - une orientation fort différente de celle conduite en centrale. Une telle différence a pu être au fil des ans source de tensions, d'autant plus difficiles à résoudre que les ethnologues de la Mission n'ont que rarement pris des postes en Drac, et réciproquement. Ainsi s'est développé petit à petit un discours critique sur ce qu'il fut convenu d'appeler la «dérive universitaire» de la Mission du patrimoine ethnologique, dérive dont le présent projet d'histoire administrative ne manquera pas d'apparaître comme un fruit d'arrière-saison. Cette étude de l'implantation de l'ethnologie en région est d'autant plus nécessaire que c'est là, en définitive qu'ont été conduites le plus grand nombre d'actions concrètes, avec une grande diversité d'orientations et une grande inventivité, variant selon la personnalité et les options scientifiques du conseiller, avec un esprit d'indépendance toujours revendiqué vis-à-vis de la « centrale». 
Pour conclure, je souhaiterais présenter ici deux autres réflexions, qui ont partie liée avec l'histoire de la Mission, sans pour autant se superposer à elle. La première a trait à la position singulièrement difficile de l'ethnologie au sein du monde des institutions culturelles et patrimoniales. Il est frappant de constater que cette discipline, qui disposait de positions très fortes dans le monde muséal il y a encore quelques décennies, a vu celles-ci se réduire ou se fragiliser progressivement, sans que l'expertise ethnologique disparaisse, mais tout se passant comme si, pour se perpétuer elle était contrainte de taire son nom - du musée de l'Homme au musée du quai Branly -, sans parler des difficultés rencontrées par la Fédération des écomusées et des musées de société, ou de l'absence, toujours problématique à mes yeux, d'une spécialité "ethnologie» clairement identifiée dans le corps ou le cadre d'emploi des conservateurs. La seconde réflexion peut se résumer à l'incapacité foncière - et il faudrait presque écrire "monumentale »- de l'administration du patrimoine à se placer vis-à-vis du corps social dans un rapport qui ne privilégie pas l'objet (à conserver) mais plutôt le lien (à créer et entretenir pour transmettre). Avoir compris trop tôt que le patrimoine était moins affaire d'objets que d'attachement à ceux-ci fut, sans doute, la plus grande victoire des ethnologues, en même temps que la cause de leur perte au sein de l'administration.

\section{BIBLIOGRAPHIE}

BARBE Noël, 2013, «Isac Chiva, ethnologie et politique patrimoniale », Terrain, $\mathrm{n}^{\circ} 60$, «L'imaginaire écologique », p. 148-163. Disponible en ligne : http://journals.openedition.org/ terrain/15127 [lien valide en novembre 2018].

HOURIEZ Elisabeth, 1969, « Un nouveau type d'instrument de recherche : le répertoire numérique détaillé », La Gazette des Archives, n 64, p. 9-16. Disponible en ligne : http://www.persee.fr/doc/ gazar_0016-5522_1969_num_64_1_2056 [lien valide en mars 2018].

HOTTIN Christian, 2011, « Entre ratification et inscriptions. La mise en œuvre d'une politique du patrimoine culturel immatériel en France (2006-2010) », Terrain, n 57, « Mentir », p. 144-157. Disponible en ligne, http://journals.openedition.org/terrain/14390 [lien valide en septembre 2018].

HOTTIN Christian, 2016a, « L'ethnologie, un métier du patrimoine ? Réflexions autour de la question du patrimoine culturel immatériel », In Situ [en ligne], n³0, « Au regard des métiers du patrimoine », http://insitu.revues.org/13633 [lien valide en septembre 2018].

ноттіN Christian, 2016b, « L'article 55. Dix ans de mise en œuvre de la Convention pour la sauvegarde du patrimoine culturel immatériel », Patrimoines. Revue de l'Institut national du patrimoine, $\mathrm{n}^{\circ} 12$, p. 20-26.

HOTTIN Christian, 2017a, «Présent et devenir du patrimoine immatériel », Le Débat, n 194, p. 147-152.

ноттіN Christian, 2017b, « Des formes de continuité du patrimoine ethnologique : ethnologie des territoires, ethnologie du patrimoine, patrimoine culturel immatériel », In Situ [en ligne], $\mathrm{n}^{\circ}$ 33, 
« Patrimoine culturel immatériel et institutions patrimoniales », http://insitu.revues.org/15469

[lien valide en septembre 2018].

RAUTENBERG Michel, 2008, « Mais pourquoi n'avance-t-on donc pas ? Le surplace de la "professionnalisation" chez les ethnologues ", in RAVIS-GIORDANI Georges (dir.). Ethnologie(s). Nouveaux contextes, nouveaux objets, nouvelles approches, Paris, Éditions du CTHS, coll. « Le regard de l'ethnologue », 2008, p. 83-99.

TORNATORE Jean-Louis, 2004, « La difficile politisation du patrimoine ethnologique », Terrain, $\mathrm{n}^{\circ} 42$, « Homme / femme », p. 149-160. Disponible en ligne, http://journals.openedition.org/ terrain/1791 [lien valide en septembre 2018].

\section{NOTES}

1. Voir en particulier les derniers de ces textes qui constituent un bilan de l'action menée entre 2006 et 2016 (Hottin 2016a, 2016b, 2017a, 2017b).

2. Quoique Christine Langlois, alors directrice de la revue Terrain, ait qualifié d'article de « témoignage » ma contribution de 2009 sur la politique française du PCI (Hottin 2011), ce qui me semble relever d'une erreur flagrante d'appréciation de ce qui constitue l'originalité de la posture du témoin, soit la prise de parole à la première personne.

3. Il s'agit du surnom donné à deux instances chargées de réduire les dépenses publiques en éliminant les administrations inutiles ou obsolètes.

\section{RÉSUMÉS}

Dans le prolongement de réflexions antérieures, cet article revient sur l'histoire de la Mission du patrimoine ethnologique en adoptant deux points de vue complémentaires. Dans un premier temps, adoptant la posture du témoin, on évoquera les évolutions de cette administration au cours des années 2000 , notamment dans le contexte de la Revue générale des politiques publiques (RGPP) au sein de la direction de l'Architecture et du Patrimoine (Dapa) du ministère de la Culture - où sera également abordée la question des prémices de la mise en œuvre de la Convention de l'Unesco pour la sauvegarde du patrimoine culturel immatériel (PCI). Dans un second temps, on proposera, de manière plus prospective, diverses pistes de recherche pour l'écriture d'une histoire administrative de la Mission du patrimoine ethnologique, depuis sa création au début des années 1980 jusqu'à l'incorporation de ses missions dans le département du Pilotage de la recherche et de la Politique scientifique (DPRPS) de la direction générale des Patrimoines: histoire des hommes et des lieux, recueil de témoignages, histoire budgétaire, analyse des tensions et des complémentarités entre services déconcentrés et administration centrale.

Pursuing earlier texts, this article takes another look at the history of the Ethnological Heritage Mission at the French Ministry of Culture, proposing two complementary points of view. To begin with, and adopting the point of view of a witness, it will take a look at the evolutions of this mission during the years after 2000, in the context of the Government's General Review of Public Policies (RGPP) and the way this affected the mission within the Architecture and Heritage 
directorate at the Ministry. This section will also examine the early stages of the implementation of the 2003 Unesco Convention on intangible heritage. In a second section, and in a more prospective framework, we will suggest some lines of enquiry that might underpin the writing of an administrative history of the Ethnological Mission, from its creation at the beginning of the 1980s up until the integration of its activities into a new department responsible for the heritage directorate's research and scientific policies. This is a history of people and places, a collection of testimonies, a budgetary history and an analysis of the tensions and complementarities between the central administration and the decentralised ethnological services in the regions.

\section{INDEX}

Keywords : administrative history, French Ministry of Culture, ethnology, ethnological heritage, intangible cultural heritage, ethnological heritage mission

Mots-clés : histoire administrative, ministère de la Culture, ethnologie, patrimoine ethnologique, patrimoine culturel immatériel, Mission du patrimoine ethnologique

\section{AUTEUR}

\section{CHRISTIAN HOTTIN}

Directeur des études de l'Institut national du patrimoine (INP), membre associé du Laboratoire d'anthropologie et d'histoire de l'institution de la culture (Lahic, IIAC, CNRS-EHESS) 